\title{
HYDRODYNAMIC STABILITY OF RAYLEIGH-BÉNARD CONVECTION WITH CONSTANT HEAT FLUX BOUNDARY CONDITION
}

\author{
BY
}

\author{
H. PARK AND L. SIROVICH
}

\section{Brown University, Providence, Rhode Island}

\begin{abstract}
We study the onset of thermal instability with the heat flux prescribed on the fluid boundaries. Assuming Boussinesq fluid, the Landau equation, which describes the evolution of the amplitude of the convection cells, is derived using the small amplitude expansion technique. For the case of a three-dimensional rectangular box with aspect ratio $(8,4,1)$, the incipient convection cell is a two-dimensional one at $P r=0.72$, which is confirmed by the numerical solution of the three-dimensional Boussinesq equation with a Chebyshev-Fourier pseudospectral code. The secondary bifurcation gives rise to an oscillatory two-dimensional roll for the same Prandtl number at $R=2.0 R_{c}$ and the motion becomes three dimensional at $R=2.8 R_{c}$.
\end{abstract}

I. Introduction. The convective instability of a layer of Boussinesq fluids heated uniformly from below is one of the simplest examples of hydrodynamic instability. This phenomenon, known as Rayleigh-Bénard (R-B) convection, shows a clear transition from the uniform conduction solution, to a steady motion varying periodically in space. It has been studied extensively due to its appearance in many fields of science and engineering. A full account of the linearized theory is given in Chandrasekhar [2] (see also Drazin and Reid [3]). Later, investigations consider nonlinear aspects of the instability, especially as a route to understanding thermal chaos and turbulence. The first work in this direction was done by Malkus and Veronis [7] and generalized by Schlüter, Lortz, and Busse [10], and many others. These analytic works on nonlinear effects were based on a perturbation expansion (basically the Poincaré-Lindstedt method) and therefore their validity is usually confined to a weak nonlinear (small amplitude) range. For the analysis of higher Rayleigh number flow, the only feasible approach appears to be numerical methods (Sirovich, Tarman, and Maxey [11], Sirovich and Park [12]).

Of prime importance in the analysis of Rayleigh-Benard convection are the boundary conditions in the vertical direction. (Periodic boundary conditions in the horizontal direction are often assumed in theoretical and computational considerations.) The usual boundary conditions adopted are stress free momentum boundary conditions (called free) or nonslip momentum boundary conditions (called rigid), and 
Dirichlet energy boundary conditions (conducting). The effects of different momentum boundary conditions have been studied rather extensively and it is generally known that differences in momentum boundary conditions do not yield drastic changes in convection pattern, but mainly change the critical Rayleigh number. The higher critical Rayleigh number for the case of rigid boundary conditions (Chandrasekhar [2]) can be understood from the fact that rigid boundary conditions are more restrictive than free boundary conditions, i.e., a smaller class of motions can be activated in the case of rigid boundary conditions under the same physical conditions. Interesting differences, quantitative as well as qualitative, appear through the change of thermal boundary conditions. The usual conducting thermal boundary condition, where we impose zero fluctuating temperature at the bounding walls, is a good approximation when the thermal conductivity of the container $\left(k_{c}\right)$ is far larger then that of the fluid $\left(k_{f}\right)$. In the other limit, $k_{f}>>k_{c}$, the appropriate thermal boundary condition is the insulating condition; that is, the normal gradient of fluctuating temperature at the boundaries is zero. This case has been considered by Jakeman [4], Riahi and Busse [9], and Sparrow, Goldstein, and Jonsson [13] who also consider more general cases. Based on experience with momentum boundary conditions one expects that the critical Rayleigh number be lower for the case of insulating thermal boundary conditions. The physical explanation is that in the case of conducting boundary conditions, a fluctuation of temperature carried to the boundary soon relaxes through the container wall, whereas at an insulating boundary it is rejected back into the fluid, and a quiescent initial linear temperature profile is more easily distorted (Normand et al., [8]). On the other hand, a lowering of the critical gradient yields a smaller energy input to the fluid, and to accommodate this decrease in energy input, the fluid develops convective cells of larger wavelength. In fact, Jeffreys [5], and later Jakeman [4], confirmed that the critical wavelength of instability becomes infinite for the insulating boundary condition.

In the present work, we reconsider the stability theory of the free-insulating boundary condition following the method used in [2]. Then the Landau equation governing the amplitude of the convection cell just above the critical Rayleigh number is derived using the small amplitude expansion technique (Schlüter, Lortz, and Busse, [10]) to determine the magnitude of the amplitude of the convection cell. Special consideration is given to the case of large aspect ratio. These results are compared with the numerical results obtained from the Chebyshev-Fourier pseudospectral method. With the additional choice of free momentum boundary conditions we obtain chaotic fluid flow at significantly lower Rayleigh numbers (therefore, requiring fewer grid points in a numerical computation). The analysis of chaotic flow for this system will be presented in a subsequent paper.

II. Stability analysis: linear theory. The system under consideration is the usual Boussinesq fluid (Drazin and Reid [3]). If we consider perturbations from the basic conduction solution, then these satisfy

$$
\begin{gathered}
\nabla \cdot \mathbf{u}=0 \\
\frac{\partial \mathbf{u}}{\partial t}+\mathbf{u} \cdot \nabla \mathbf{u}=-\nabla P+R \operatorname{Pr} \theta \mathbf{k}+\operatorname{Pr} \Delta \mathbf{u},
\end{gathered}
$$




$$
\begin{gathered}
\frac{\partial \theta}{\partial t}+\mathbf{u} \cdot \nabla \theta=w+\Delta \theta \\
\mathbf{u}=(u, v, w),
\end{gathered}
$$

with the following boundary conditions:

$$
z=0,1 ; \quad \frac{\partial u}{\partial z}=\frac{\partial v}{\partial z}=w=\frac{\partial \theta}{\partial z}=0 .
$$

Here we adopted the standard normalization

$$
\begin{aligned}
& x=x^{*} / d, \quad t=k t^{*} / d^{2}, \quad u=d u / k, \\
& \theta=\theta^{*} / \beta d, \quad p=d^{2} p^{*} / \rho_{0} k^{2},
\end{aligned}
$$

where dimensional variables are denoted by an asterisk. We let $\beta d \equiv \theta_{0}-\theta_{1}$ (temperature difference between two bounding walls), the Rayleigh number $R \equiv$ $g \alpha \beta d^{4} / k \nu$, and the Prandtl number $\operatorname{Pr} \equiv \nu / k$.

Following [2], we assume

$$
\begin{gathered}
w=W(z) f(x, y) e^{s t}, \\
\theta=T(z) f(x, y) e^{s t},
\end{gathered}
$$

where the planform function $f(x, y)$ satisfies $\nabla_{\perp}^{2} f=-a^{2} f$, and neglecting nonlinear terms we find

$$
\begin{gathered}
\left(D^{2}-a^{2}\right)\left(D^{2}-a^{2}-s / P r\right) W=a^{2} R T, \\
\left(D^{2}-a^{2}-s\right) T=-W .
\end{gathered}
$$

Since the exchange of stability is valid for R-B with symmetric boundary conditions $[2,3]$, we may put $s=0$ a priori and get a single equation for $T$,

$$
\left(D^{2}-a^{2}\right)^{3} T=-a^{2} R T,
$$

with boundary conditions

$$
z=0,1 ; \quad D T=0, \quad\left(D^{2}-a^{2}\right) T=0, \quad D^{2}\left(D^{2}-a^{2}\right) T=0 .
$$

A solution of $(2.11)$ has the form

$$
T=e^{q z}
$$

The roots of Eq. (2.12) are found to be

$$
q= \pm i q_{0}, \pm\left(q_{1}+i q_{2}\right), \pm\left(q_{1}-i q_{2}\right),
$$

where

$$
\begin{aligned}
q_{0} & =a(\tau-1)^{1 / 2} \\
q_{1,2} & =a\left\{\frac{1}{2} \sqrt{1+\tau+\tau^{2}}+\frac{1}{2}\left(1 \pm \frac{1}{2} \tau\right)\right\}^{1 / 2} \\
\tau & =\left(R / a^{4}\right)^{1 / 3}
\end{aligned}
$$

From symmetry of the problem the proper solution of Eq. (2.11) is either even or odd in $z$. From general considerations, the lowest state will be even with no nodes [2]. Therefore we consider the even solution.

$$
T(z)=A_{0} \cos q_{0} z+A \cosh q z+A^{*} \cosh q^{*} z,
$$




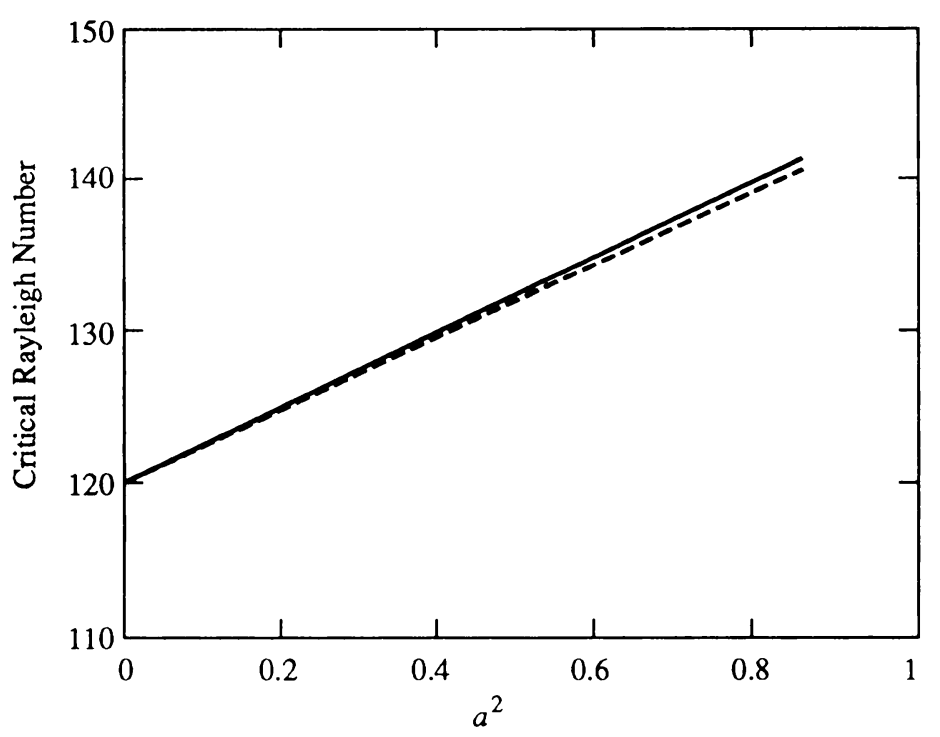

FIG. 2.1. Critical Rayleigh number $R_{c}$ as a function of wave number squared $a^{2} ;$ - exact result; - - asymptotic result $\left(a^{2} \rightarrow 0\right)$.

where $q^{*}=q_{1}+i q_{2}$. After imposing boundary conditions we obtain the following characteristic equations to determine the critical Rayleigh number:

$$
q_{0} \tan \frac{q_{0}}{2}-\frac{2 q_{1} \sinh q_{1}-2 q_{2} \sin q_{2}}{\cosh q_{1}+\cos q_{2}}=0 .
$$

The solution of this algebraic equation yields the minimum critical Rayleigh number at $a=0$ (cf. Fig. 2.1)

$$
R_{c}=120 \text {. }
$$

The low value of $R_{c}$ and the vanishing value of $a_{c}$ are caused by the fact that many modes can be activated with this least restrictive boundary condition and a convective cell of large wavelength is developed to accommodate a smaller energy input.

III. Nonlinear theory. Linear theory determines the critical Rayleigh number but does not say anything about the magnitude of amplitude finally obtained. Generally, it is known that the amplitude of the convection velocity increases, near the onset of convection, according to the power law

$$
(\mathbf{u}, \theta)=O(\sqrt{\varepsilon}),
$$

where

$$
\varepsilon \equiv\left(R-R_{c}\right) / R_{c} .
$$

This follows from the small amplitude perturbation technique (Malkus and Veronis [7], Schlüter, Lortz, and Busse [10]).

For the analysis of the weakly nonlinear problem, we expand in powers of $\varepsilon$,

$$
\mathbf{u}=\varepsilon \mathbf{u}_{1}+\varepsilon^{2} \mathbf{u}_{2}+\varepsilon^{3} \mathbf{u}_{3}+\cdots
$$




$$
\begin{gathered}
\theta=\varepsilon \theta_{1}+\varepsilon^{2} \theta_{2}+\varepsilon^{3} \theta_{3}+\cdots, \\
R=R_{0}+\varepsilon^{2} R_{2}+\cdots,
\end{gathered}
$$

and scale the time $t$ as $\partial_{t} \equiv \varepsilon^{2} \partial_{\tau}$. (The linear term in (3.5) vanishes by symmetry.) After substituting these expansions into (2.2) and (2.3), we find the following sequence of linear inhomogeneous equations (see [10]).

Order $\varepsilon$ :

$$
\begin{gathered}
\left(D^{2}-a^{2}\right)^{2} h-R_{c} g=0, \\
\left(D^{2}-a^{2}\right) g+a^{2} h=0,
\end{gathered}
$$

where

$$
\begin{gathered}
\mathbf{u}=\left(\nabla \frac{\partial}{\partial z}-\mathbf{e}_{z} \nabla^{2}\right) f(x, y) h(z), \\
\theta=f(x, y) g(z),
\end{gathered}
$$

and

$$
\nabla_{\perp}^{2} f=-a^{2} f .
$$

$R_{c}$ is the critical Rayleigh number. This analysis of linear stability implies that we consider the stability of the $\cos a x$ mode,

$$
f=A \cos a x .
$$

Since the critical wavenumber $a_{c}$ vanishes, in the numerical investigation that follows we consider a rectangular region and thus anticipate that the convection pattern just above the critical Rayleigh number will be two-dimensional roll parallel to the shorter horizontal direction. The numerical solution which is fully three dimensional does confirm this.

The appropriate boundary conditions are

$$
z=0,1 ; \quad h=D^{2} h=D g=0 .
$$

Order $\varepsilon^{2}$ :

$$
\left[\begin{array}{cc}
\nabla^{4} & R_{c} \nabla_{1}^{2} \\
1 & \nabla^{2}
\end{array}\right]\left[\begin{array}{c}
W_{2} \\
\theta_{2}
\end{array}\right]=\left[\begin{array}{c}
-\frac{1}{P r} A^{2} a^{4}(\cos 2 a x)\left(h^{\prime} h^{\prime \prime}-h h^{\prime \prime \prime}\right) \\
A^{2} a^{2}\left(\frac{h^{\prime} g+h g^{\prime}}{2}\right)+A^{2} a^{2}(\cos 2 a x)\left(\frac{h g^{\prime}-h^{\prime} g}{2}\right)
\end{array}\right]
$$

Here $W_{2}$ is the $z$-component velocity of $\mathbf{u}_{2}$.

To solve (3.13) we write

$$
\begin{gathered}
W_{2}=A^{2}(\cos 2 a x) W_{2}^{\mathrm{II}}(z), \\
\theta_{2}=A^{2} \theta_{2}^{\mathrm{I}}(z)+A^{2}(\cos 2 a x) \theta_{2}^{\mathrm{II}}(z) .
\end{gathered}
$$

Equation (3.13) can then be rewritten as

$$
D^{2} \theta_{2}^{1}=a^{2}\left[\frac{h^{\prime} g+h g^{\prime}}{2}\right]
$$




$$
\begin{gathered}
{\left[D^{2}-(2 a)^{2}\right]^{2} W_{2}^{\mathrm{II}}+R_{c}\left[-(2 a)^{2}\right] \theta_{2}^{\mathrm{II}}=\frac{1}{P r} a^{4}\left[h h^{\prime \prime \prime}-h^{\prime} h^{\prime \prime}\right],} \\
{\left[D^{2}-(2 a)^{2}\right] \theta_{2}^{\mathrm{II}}+W_{2}^{\mathrm{II}}=a^{2}\left[\frac{h g^{\prime}-h^{\prime} g}{2}\right]}
\end{gathered}
$$

The corresponding boundary conditions are

$$
z=0,1 ; \quad W_{2}^{\mathrm{II}}=D^{2} W_{2}^{\mathrm{II}}=D \theta_{2}^{\mathrm{I}}=D \theta_{2}^{\mathrm{II}}=0
$$

An arbitrary constant arising from the solution of Eq. (3.16) due to the Neumann boundary condition is fixed by the following normalization condition which is set up for later convenience:

$$
\left\langle\theta_{1} \theta_{2}\right\rangle=0
$$

Here $\langle\cdot\rangle$ means the volume average.

Order $\varepsilon^{3}$ :

$$
\begin{aligned}
& \left(D^{2}-a^{2}\right)^{2} \Phi_{3}-R_{c} a^{2} \psi_{3}=\frac{1}{\operatorname{Pr}} \frac{\partial A}{\partial t}\left(a^{2} h^{\prime \prime}-a^{4} h\right)+a^{2} R_{2} A g \\
& -\frac{1}{2} \frac{1}{P r} A^{3}\left[-a^{2} h^{\prime}\left(D^{2} W_{2}^{\mathrm{II}}\right)+a^{2} h^{\prime \prime \prime} W_{2}^{\mathrm{II}}+3 a^{4} h^{\prime} W_{2}^{\mathrm{II}}\right. \\
& \left.-\frac{a^{2}}{2} h D^{3} W_{2}^{\mathrm{II}}+\frac{a^{2}}{2}\left(D W_{2}^{\mathrm{II}}\right) h^{\prime \prime}+\frac{3}{2} a^{4} h\left(D W_{2}^{\mathrm{II}}\right)\right],
\end{aligned}
$$

$$
\begin{aligned}
\left(D^{2}-a^{2}\right) \psi_{3}+\Phi_{3}= & \frac{\partial A}{\partial t} g+\frac{A^{3}}{2}\left[2 a^{2} h^{\prime} \theta_{2}^{\mathrm{II}}+\frac{1}{2} g D W_{2}^{\mathrm{II}}+a^{2} h D \theta_{2}^{\mathrm{II}}+g^{\prime} W_{2}^{\mathrm{II}}\right] \\
& +A^{3} a^{2} h\left(D \theta_{2}^{\mathrm{I}}\right)
\end{aligned}
$$

where $\Phi_{3}(z)$ and $\psi_{3}(z)$ arise from

$$
\begin{aligned}
& W_{3}=\Phi_{3} \cos a x, \\
& \theta_{3}=\psi_{3} \cos a x,
\end{aligned}
$$

and $\mathbf{u}_{3}=\left(u_{3}, v_{3}, W_{3}\right)$. 
If we apply solvability conditions to Eq. (2.35) and (2.36) we obtain the following amplitude equation (the Landau equation):

$$
\begin{aligned}
\frac{\partial A}{\partial t}\left[\int_{0}^{1}\right. & \left.\frac{1}{P r}\left(-a^{2} h^{\prime \prime}+a^{4} h\right) a^{2} h d z+\int_{0}^{1}\left(R_{c} a^{2}\right) g^{2} d z\right] \\
= & R_{2} A\left[\int_{0}^{1}\left(a^{2} g\right)\left(a^{2} h\right) d z\right] \\
+ & A^{3}\left[\int _ { 0 } ^ { 1 } ( - \frac { 1 } { 2 } \frac { 1 } { P r } ) \left\{-a^{2} h^{\prime}\left(D^{2} W_{2}^{\mathrm{II}}\right)+a^{2} h^{\prime \prime \prime} W_{2}^{\mathrm{II}}+3 a^{4} h^{\prime} W_{2}^{\mathrm{II}}\right.\right. \\
& -\frac{a^{2}}{2} h\left(D^{3} W_{2}^{\mathrm{II}}\right)+\frac{a^{2}}{2}\left(D W_{2}^{\mathrm{II}} h^{\prime \prime}+\frac{3}{2} a^{4} h\left(D W_{2}^{\mathrm{II}}\right\}\left(a^{2} h\right)\right. \\
+ & \frac{1}{2}\left(-R_{c} a^{2}\right)\left\{2 a^{2} h^{\prime} \theta_{2}^{\mathrm{II}}+\frac{1}{2} g D W_{2}^{\mathrm{II}}+a^{2} h D \theta_{2}^{\mathrm{II}}+g^{\prime} W_{2}^{\mathrm{II}}\right\} g \\
& \left.+\left(-R_{c} a^{2}\right)\left\{a^{2} h\left(D \theta_{2}^{\mathrm{I}}\right)\right\} g d z\right] .
\end{aligned}
$$

Therefore the magnitude of the amplitude of the convection cell is given by

$$
A^{2}=-R_{2} \sigma / \gamma
$$

where $\sigma$ is the coefficient of the linear term and $\gamma$ is the coefficient of the cubic term of the Landau equation (3.25). To give the amplitude, $A$, physical meaning we relate it to $\sqrt{\left\langle\theta^{2}\right\rangle}$. The mean square fluctuating temperature, $\sqrt{\left\langle\theta^{2}\right\rangle}$, can be obtained in the following way. Since we defined

we have

$$
\begin{aligned}
& \theta=\varepsilon \theta_{1}+\varepsilon^{2} \theta_{2}+\cdots, \\
& R=R_{0}+\varepsilon^{2} R_{2}+\cdots,
\end{aligned}
$$

$$
\left\langle\theta^{2}\right\rangle=\varepsilon^{2}\left\langle\theta_{1}^{2}\right\rangle+\varepsilon^{3}\left\langle\theta_{1} \theta_{2}\right\rangle+O\left(\varepsilon^{4}\right)
$$

where

$$
\theta_{1}=A g \cos a x
$$

But $\left\langle\theta_{1} \theta_{2}\right\rangle$ is zero due to the normalization condition (3.20), which was introduced to fix arbitrary constants in the eigenvalue problem. Substitution of (3.4) and (3.29) into (3.28) yields the final expression

$$
\left\langle\theta^{2}\right\rangle=\left(-\frac{\beta}{\gamma}\right)\left(R-R_{c}\right) \frac{1}{2} \int_{0}^{1} g^{2} d z=c A^{2} .
$$

In principle we can use the analytic solution for $h(z)$ and $g(z)$ obtained in Sec. II (e.g., Eq. (2.16)) to get the numerical values of the coefficients $\sigma$ and $\gamma$ of the Landau equation (3.25). But to avoid massive algebraic manipulation, we have integrated all the above sets of linear equations numerically. In the case of small $a^{2}$, the analytic integration can be done more easily and explicit forms for $g$ and $h$ appear. (See below and the Appendix.) 
Asymptotic derivation of the Landau equation. The critical wavenumber $a$ for a finite box is given by

$$
a=\frac{2 \pi}{\max \left(L_{x}, L_{y}\right)}
$$

for the case of heat flux boundary conditions. For a shallow box, where the horizontal length is far larger than the vertical length, $a$ is a small value and we derive the Landau equation relatively easily. The details of derivation are presented in the Appendix. Here we show only the summary of the results.

Solving $O(\varepsilon)$ equations (linear stability problem) by a regular perturbation method, using $a^{2}$ as a small parameter, we get the critical Rayleigh number in terms of $a^{2}$

$$
R_{c}=120+23.6147 a^{2} .
$$

We plot Eq. (3.33) in comparison with the exact value of $R_{c}$ in Fig. 2.1. After solving $O\left(\varepsilon^{2}\right)$ and $O\left(\varepsilon^{3}\right)$ equations in a similar fashion, we find finally the Landau equation and root mean square of the temperature fluctuation:

$$
\frac{d}{d \tau}\left(C_{g} A\right)=\frac{R_{2}^{(1)}}{2 \cdot 3 \cdot 4 \cdot 5}\left(C_{g} A\right)-\frac{3}{2} \cdot \frac{25}{2} \cdot \frac{31}{9 \cdot 7 \cdot 5 \cdot 2}\left(C_{g} A\right)^{3}
$$

and

$$
\sqrt{\left\langle\theta^{2}\right\rangle}=\sqrt{\Delta R(0.0045161) / a^{2}}
$$

where we may take $C_{g}=1$ without loss of generality and $\Delta R \equiv R-R_{c}$. The expressions (3.34) and (3.35) are valid up to $a^{2}$ and are expected to give accurate results when $a^{2}$ is small.

IV. Numerical solution of the Boussinesq equation. Next we numerically solve the full three-dimensional Boussinesq equation with boundary condition (2.5) in order to confirm the analytic results and to examine the transition from steady convection to time dependent convection (oscillatory instability). For this purpose governing equations are rewritten in rotational form,

$$
\begin{gathered}
\nabla \cdot \mathbf{u}=0, \\
\frac{\partial}{\partial t} \mathbf{u}=-\nabla\left(\frac{p}{\rho}+\frac{1}{2} \mathbf{u} \cdot \mathbf{u}\right)+\mathbf{u} \wedge(\nabla \wedge \mathbf{u})+\operatorname{Pr} \nabla^{2} \mathbf{u}+\operatorname{RPr} \theta \mathbf{e}_{z}, \\
\frac{\partial \theta}{\partial t}=-\nabla \cdot(\mathbf{u} \theta)+\nabla^{2} \theta+w,
\end{gathered}
$$

with the boundary conditions

$$
z= \pm 1 ; \quad \frac{\partial u}{\partial z}=\frac{\partial v}{\partial z}=w=\frac{\partial \theta}{\partial z}=0 .
$$

Here we changed the domain from $[0,1]$ to $[-1,1]$ for convenience of numerical solution.

These equations with the corresponding boundary conditions are solved by a pseudospectral method with the influence matrix technique (Canuto, Hussaini, Quateroni, 
and Zang [1], Kleiser and Schumann [6]). The numerical method is based on Fourier expansion in the horizontal directions,

$$
\begin{aligned}
& \mathbf{u}(\mathbf{x}, t)=\sum_{k_{1}} \sum_{k_{2}} \overline{\mathbf{u}}\left(k_{1}, k_{2}, x_{3}, t\right) \exp \left(i k_{1} x_{1}\right) \exp \left(i k_{2} x_{2}\right), \\
& \theta(\mathbf{x}, t)=\sum_{k_{1}} \sum_{k_{2}} \bar{\theta}\left(k_{1}, k_{2}, x_{3}, t\right) \exp \left(i k_{1} x_{1}\right) \exp \left(i k_{2} x_{2}\right),
\end{aligned}
$$

and Chebyshev expansion of the Fourier coefficients $\overline{\mathbf{u}}$ and $\bar{\theta}$ for each value of $k_{1}$ and $k_{2}$,

$$
\begin{aligned}
& \overline{\mathbf{u}}\left(k_{1}, k_{2}, x_{3}, t\right)=\sum_{k_{3=0}}^{N_{3}} \hat{\mathbf{u}}\left(k_{1}, k_{2}, k_{3}, t\right) T_{k 3}\left(x_{3}\right), \\
& \bar{\theta}\left(k_{1}, k_{2}, x_{3}, t\right)=\sum_{k_{3=0}}^{N_{3}} \hat{\theta}\left(k_{1}, k_{2}, k_{3}, t\right) T_{k 3}\left(x_{3}\right) .
\end{aligned}
$$

Because of our special boundary conditions (Eq. (2.5)), $\theta$ and $w(z$-component velocity) have different parities in the vertical direction and Fourier expansion cannot be used without inducing the Gibbs phenomenon. Multiplications in the nonlinear terms are performed in the physical space, while derivatives are computed in the spectral space. Transformations between physical and spectral space are done using fast Fourier transform algorithms. Time marching is carried out in the spectral space. We use a second-order Adams-Bashforth scheme for nonlinear and source terms and a Crank-Nicolson scheme for diffusion and pressure terms.

As an initial condition for the numerical integration, we impose a small random perturbation on the temperature field, viz.

$$
\theta\left(x_{i}, y_{j}, z_{k}\right)=10^{-5} \eta_{i j k},
$$

where the $\eta_{i j k}$ are random numbers in the interval $[0,1]$, and the residual net momentum in the horizontal direction was subtracted from the flow field after several time steps. The resulting flow field was adopted as an initial condition for the subsequent numerical integration. One must be careful at this step to eliminate an artificial translation of the convection cell since it is easily shown that the initial horizontal momentum is an invariant of the system.

V. Results and discussions. Our aim in this study is to investigate the flow field above the critical Rayleigh number for free-insulating boundary conditions. The Prandtl number of the fluid is taken to be 0.72 . Figure 2.1 shows the critical Rayleigh number as a function of the aspect ratio of the system (or, equivalently, wavenumber). The critical Rayleigh number with this boundary condition is lower than those with other boundary conditions because this boundary condition, free-insulating, is the least restrictive in the sense that the most modes can be activated under the same physical conditions. On the other hand, a lowering of critical gradient yields a smaller energy input to the fluid, and to accommodate this decrease in energy input, the fluid develops a convective cell of larger wavelength. 
Figures 5.1-5.4 show a summary of the numerical solution of a three-dimensional Boussinesq equation at $\operatorname{Pr}=0.72$ for the box aspect ratio of $(8,4,1)$ with the pseudospectral code. In Fig. 5.1 the streamlines of the calculated flow field at the Rayleigh number of $1.005 R_{c}$ are plotted. The structure of the convection cell is a pair of two-dimensional rolls parallel to the shorter horizontal direction. This is consistent with the linear stability analysis, since linear theory (cf. Fig. 2.1) says that the most dangerous mode is that with the minimum wavenumber when the boundary condition is insulating. The convection cells exhibit slightly vertical asymmetry. This is worth pointing out inasmuch as the cells are often drawn as vertically symmetric, but, as the equations reveal, such a symmetry is incompatible with physics (symmetric convection cells are correct only in linear theory). The profiles of the fluctuating temperature $\theta$ at various horizontal locations are displayed in Fig 5.2a. At a very small value of the Rayleigh number $\left(1.005 R_{c}\right)$, the temperature profiles are almost constants, which explains why we obtained the lowest order perturbation solution of temperature $g^{(0)}(z)=C_{g}$ in the Appendix. The horizontal variation of $\theta(z)$ from the numerical solution can be explained as the effect of the horizontal mode $\cos a x$ in the analytic (perturbation) solution. As the Rayleigh number is increased to $2.0 R_{c}$ (Fig. 5.2b), the curvature of the $\theta(z)$ profiles increases under the constraint of zero normal flux at the walls.

Figure 5.3 reveals the time variation of $\left\langle\theta^{2}\right\rangle$ and $\left\langle w^{2}\right\rangle$ at $1.002 R_{c}$ at a stationary state. The constant values of amplitude mean that the bifurcation from the conduction solution is a supercritical one. The secondary bifurcation occurs near $2.0 R_{c}$, where the amplitude of a two-dimensional roll becomes oscillatory. Figure 5.4 presents the streamlines and temporal variation of amplitudes at $2.0 R_{c}$, which shows a weak oscillation of a two-dimensional convection roll. The motion becomes three dimensional at $R=2.8 R_{c}$.

Now we compare the magnitude of the steady convection cell from numerical code with that from perturbation solutions. In Fig. 5.5 we plot the mean square average of the fluctuating temperature field $\sqrt{\left\langle\theta^{2}\right\rangle}$ from numerical results, from the exact Landau equation (solid line, cf. Eq. (3.31)), and from the asymptotic Landau equation (broken line, cf. Eq. (A.50)). For the range of $R<1.2 R_{c}$, values from numerical results show a very good agreement with those of the exact and asymptotic Landau equations. There is a small deviation between the exact Landau equation and the asymptotic Landau equation because the critical Rayleigh numbers from these two equations are slightly different.

When we rewrite the Landau equation as

$$
\alpha \frac{d A}{d t}=\sigma A-\gamma A^{3}
$$

the exact solution can be obtained as

$$
|A|^{2}=A_{0}^{2} /\left\{\frac{\gamma}{\sigma} A_{0}^{2}+\left(1-\frac{\gamma}{\sigma} A_{0}^{2}\right) e^{-2 \sigma t / \alpha}\right\},
$$

where $A_{0}$ is the initial value of $A$. Since $\sigma$ is proportional to $\Delta R\left(\equiv R-R_{c}\right)$, the growth (or the decay) rate of amplitude becomes very slow as $\Delta R$ becomes 
(a)

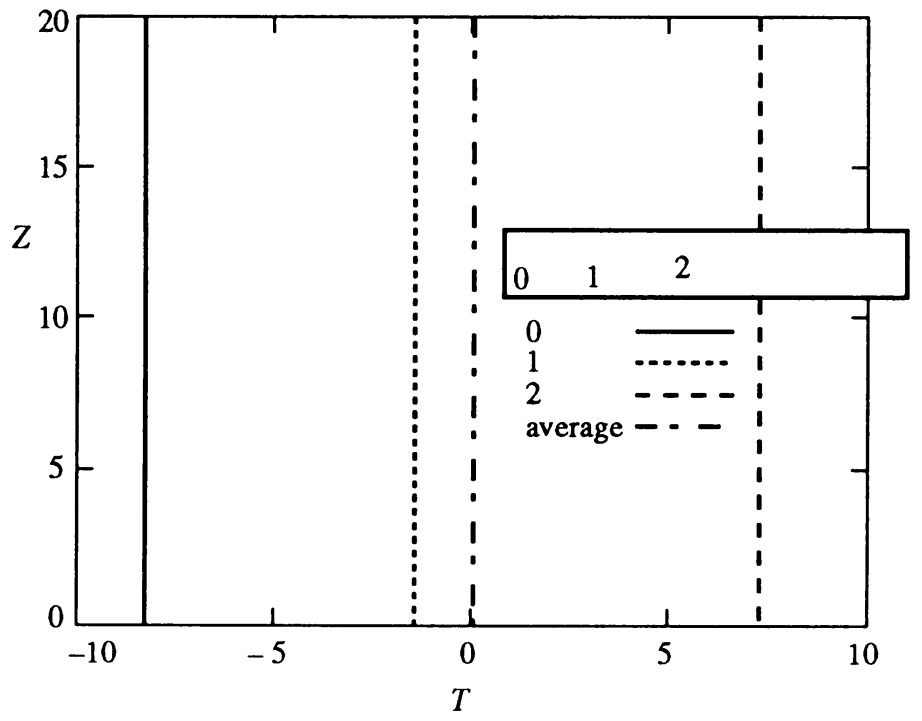

(b)

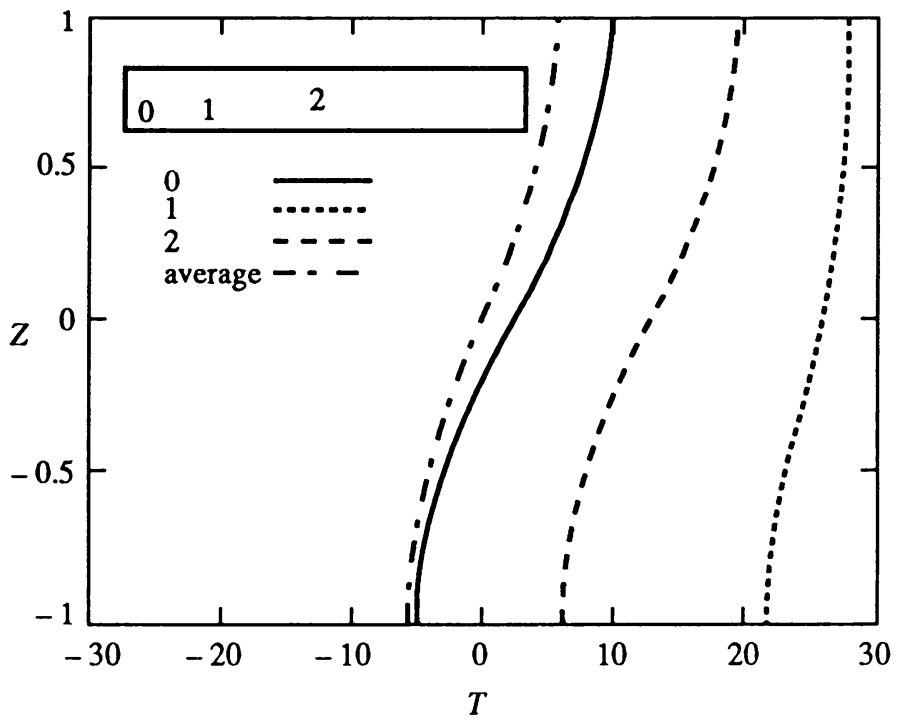

FIG. 5.2. The profiles of the fluctuating temperature $\theta$ at various horizontal locations for (a) $R=1.005 R_{c}$; (b) $R=2.0 R_{c}$. 

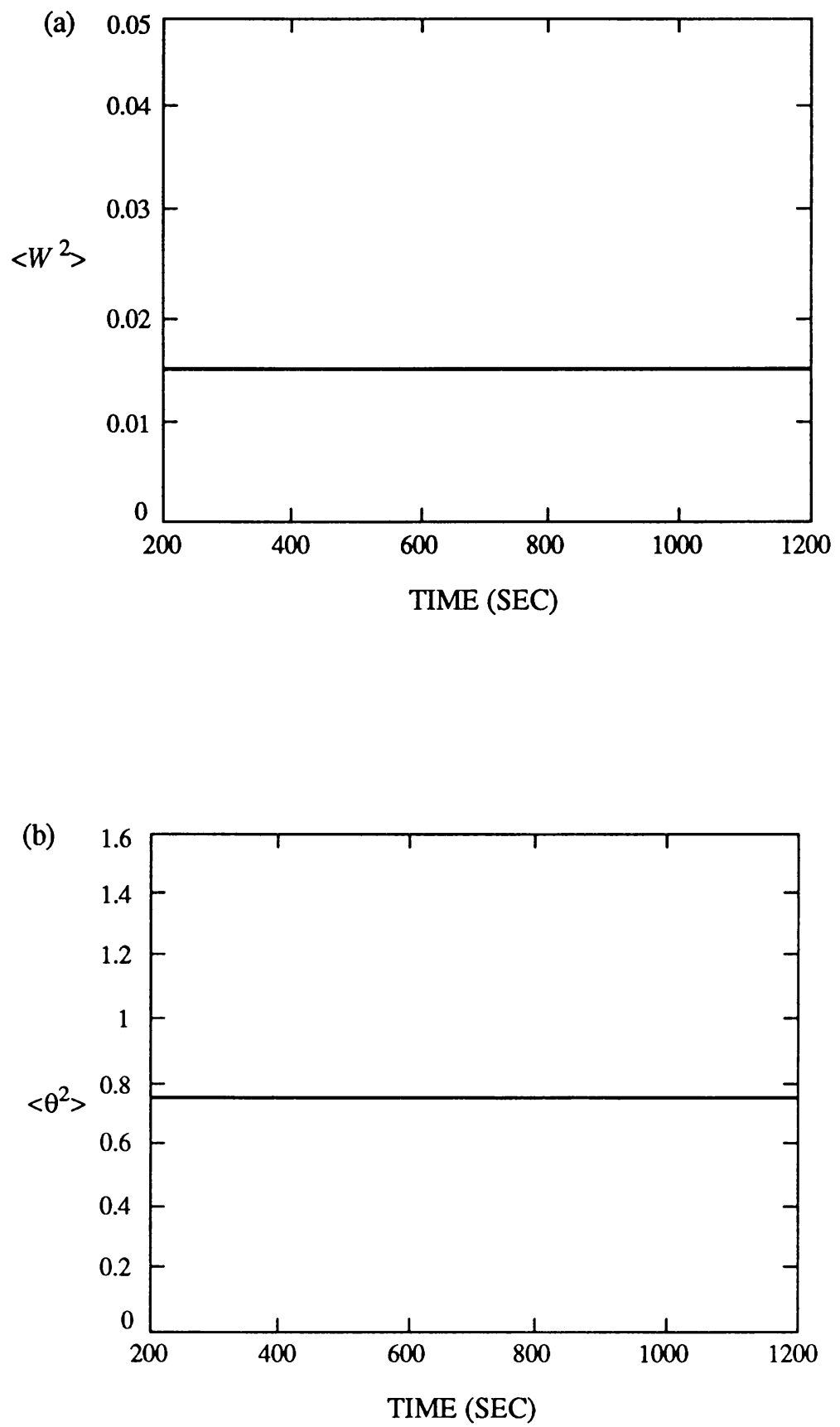

FIG. 5.3. The time variation of $\left\langle\theta^{2}\right\rangle$ and $\left\langle w^{2}\right\rangle$ at $1.002 R_{c}$. 
(a)

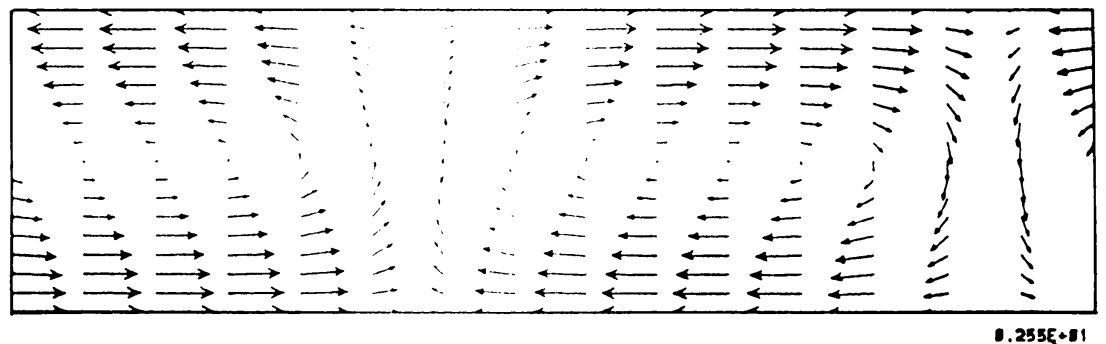

(b)
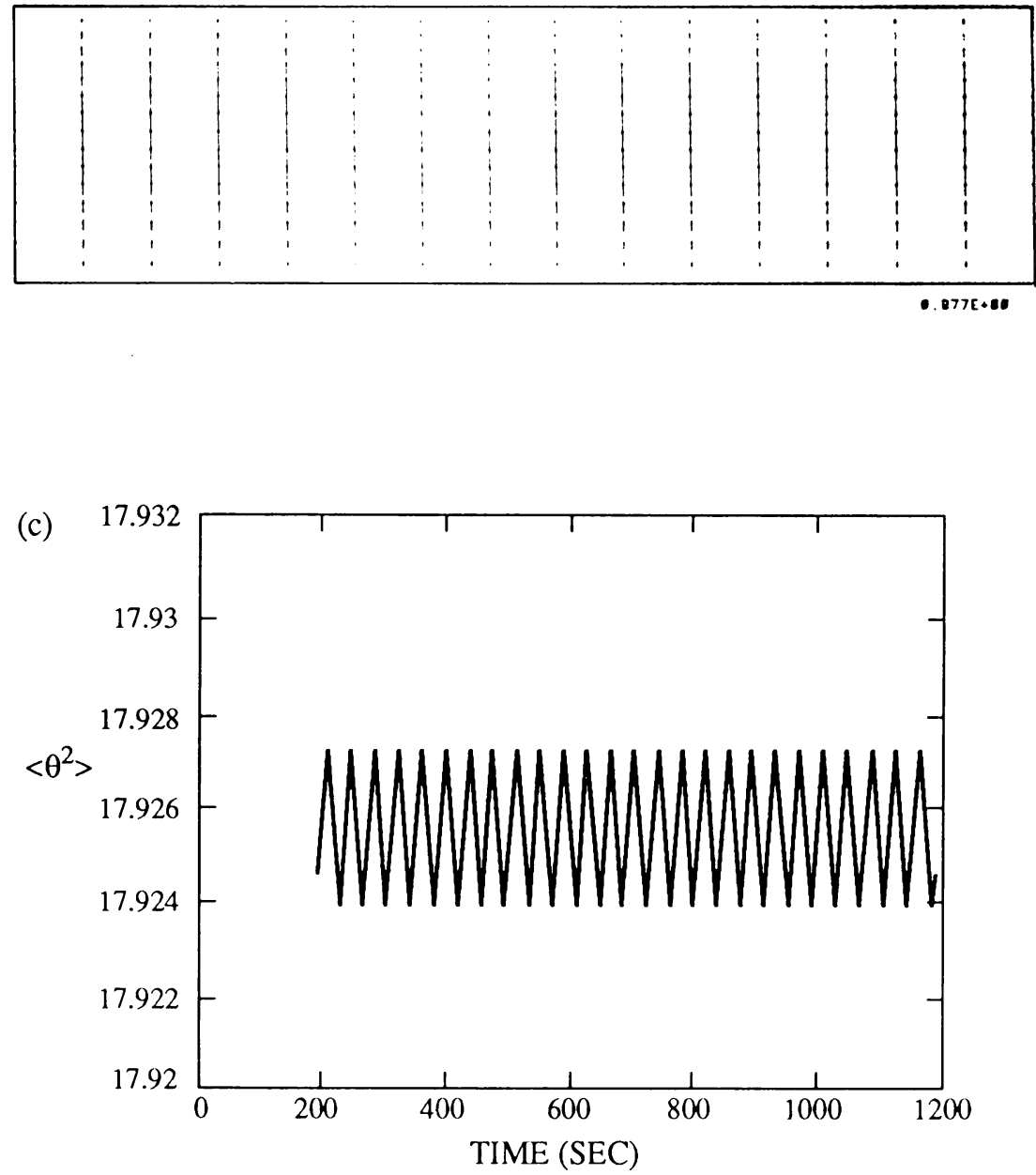

FIG. 5.4. The streamlines and the temporal variation of amplitudes at $R=2.0 R_{c}$ : (a) streamline at $x-z$ cross section; (b) streamline at $y-z$ cross section; (c) time senses of $\left\langle\theta^{2}\right\rangle$. 


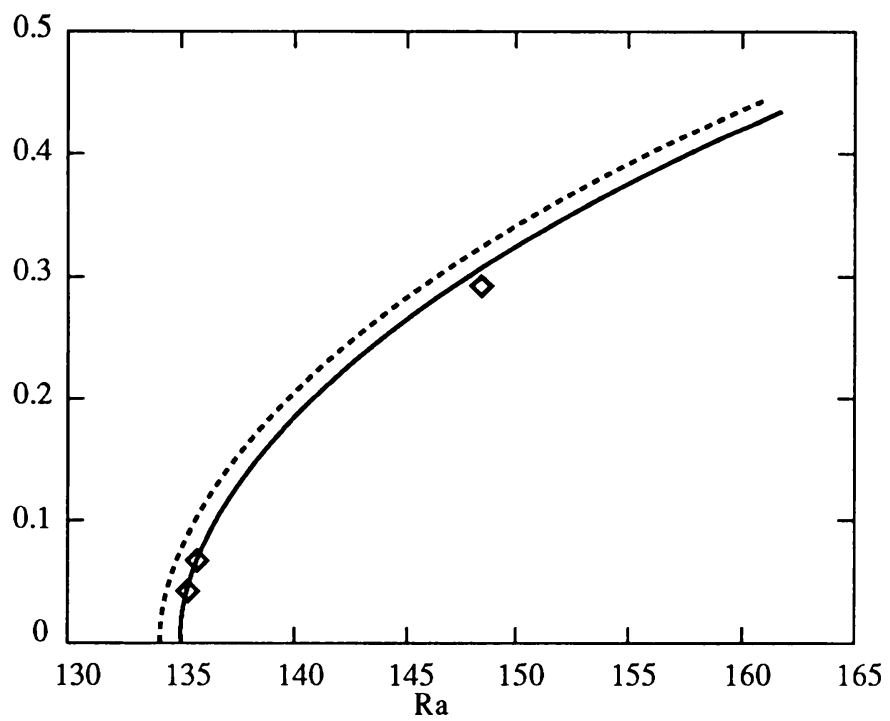

Fig. 5.5. The mean square average of the fluctuating temperature field $\sqrt{\left\langle\theta^{2}\right\rangle}$; - exact Landau equation, (3.31);- - - asymptotic Landau equation, (A.50); $\diamond \diamond$ numerical solution of Boussinesq equation.

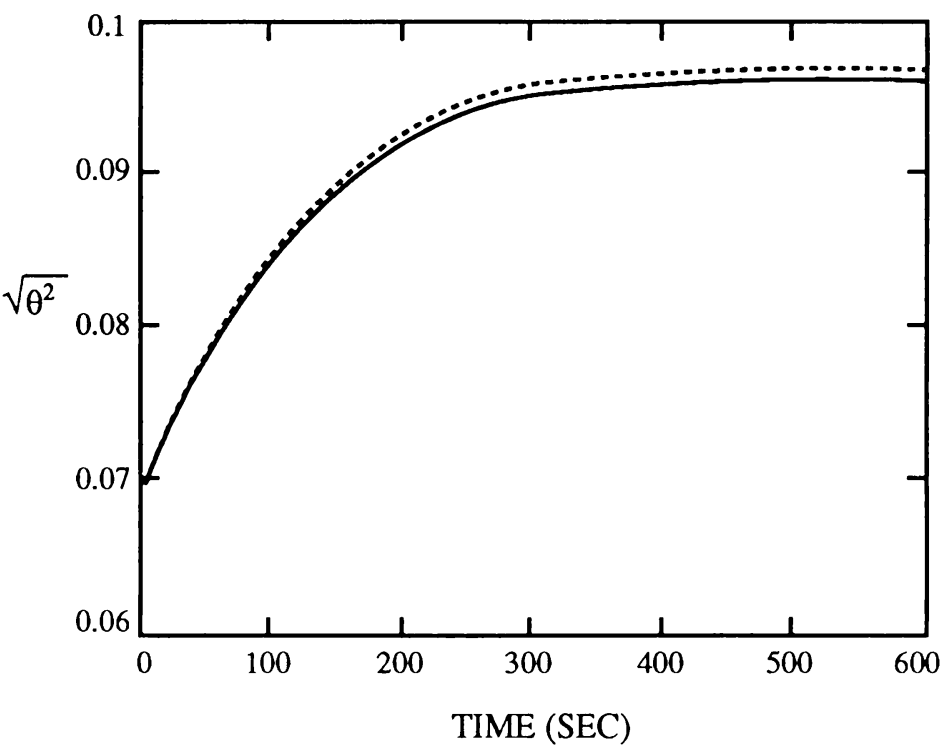

Fig. 5.6. The time dependence of $\sqrt{\left\langle\theta^{2}\right\rangle}$.

VI. Conclusion. We have derived the Landau equation from the Boussinesq equation using a small amplitude expansion technique for the case of free-insulating boundary conditions. Exploiting the fact that the critical wavenumber $a$ is a small value, we have also derived an asymptotic Landau equation which is valid up to $O\left(a^{2}\right)$. The results from these equations are compared with the results from the 
numerical solution of a three-dimensional Boussinesq equation using a ChebyshevFourier pseudospectral method. Generally these results agree with each other very well. Using the pseudospectral code, we have examined the transition from a stationary two-dimensional roll to an oscillatory two-dimensional roll (secondary bifurcation) and this phenomenon will be investigated further in a subsequent paper.

Appendix. In this appendix, we present a derivation of the Landau equation which is valid for small values of the wave number $a$.

$[O(\varepsilon)]$ : The $O(\varepsilon)$ equations were given as (3.6) and (3.7). Noting that $a^{2}$ is a small parameter, we assume

$$
\begin{gathered}
g=g^{0)}+a^{2} g^{(1)}+a^{4} g^{(2)}+\cdots, \\
h=h^{(0)}+a^{2} h^{(1)}+a^{4} h^{(2)}+\cdots, \\
R_{c}=R_{c}^{(0)}+a^{2} R_{c}^{(1)}+a^{4} R_{c}^{(2)}+\cdots .
\end{gathered}
$$

Equations (A.1), (A.2), and (A.3) are substitued into Eqs. (3.6) and (3.7) to yield the following sequences of perturbation equations. In all subsequent equations, the appropriate boundary conditions are:

$$
z=0,1 ; \quad W=D^{2} W=D \theta=0 .
$$

$$
\left\langle O\left(a^{0}\right)\right\rangle
$$

$$
\begin{gathered}
D^{2} g^{(0)}=0, \\
D^{4} h^{(0)}=R_{c}^{(0)} g^{(0)} .
\end{gathered}
$$

Then

$$
\begin{gathered}
g^{(0)}=C_{g}(\text { constant }), \\
h^{(0)}=\frac{R_{c}^{(0)} C_{g}}{4 !}\left(z^{4}-2 z^{3}+z\right) .
\end{gathered}
$$

$\left\langle O\left(a^{2}\right)\right\rangle$

$$
\begin{gathered}
D^{2} g^{(1)}-g^{(0)}+h^{(0)}=0, \\
D^{4} h^{(1)}-2 D^{2} h^{(0)}-R_{c}^{(0)} g^{(1)}-R_{c}^{(1)} g^{(0)}=0 .
\end{gathered}
$$

As before, we have to impose solvability conditions for the perturbation solution to exist. Here the homogeneous equations are

$$
\begin{aligned}
D^{2} g & =0, \\
D^{4} h & =0 .
\end{aligned}
$$

The homogeneous solutions are $g=c$ (a constant) and $h=0$. The structure of perturbation equations in the subsequent analysis takes the form

$$
\begin{aligned}
D^{2} g^{c} & =g_{i}, \\
D^{4} h^{i} & =h_{i},
\end{aligned}
$$


where the right-hand sides are known functions. Therefore the solvability condition applied to Eq. (A.12) yields

$$
\int_{0}^{1} g_{i} d z=0
$$

and the solvability condition for Eq. (A.13) is trivial. When we apply the solvability condition to (A.8), we find

$$
R_{c}^{(0)}=5 !=120 \text {. }
$$

The solutions of (A.8) and (A.9) are given by:

$$
\begin{aligned}
h^{(1)}= & 2 C_{g}\left[\frac{R_{c}^{(0)}}{2 \cdot 3 \cdot 4 \cdot 5 \cdot 6} z^{6}-\frac{R_{0}^{(0)}}{2 \cdot 2 \cdot 3 \cdot 4 \cdot 5} z^{5}\right] \\
& +R_{c}^{(0)} C_{g}\left[\frac{z^{6}}{2 \cdot 3 \cdot 4 \cdot 5 \cdot 6}-\frac{z^{10}}{6 \cdot 7 \cdot 8 \cdot 9 \cdot 10}+\frac{z^{9}}{2 \cdot 6 \cdot 7 \cdot 8 \cdot 9}\right. \\
& \left.\quad-\frac{z^{7}}{6 \cdot 4 \cdot 6 \cdot 7}-\frac{z^{4}}{2 \cdot 4 \cdot 7 \cdot 2 \cdot 3 \cdot 4}\right] \\
& +R_{c}^{(1)} C_{g} \frac{z^{4}}{2 \cdot 3 \cdot 4}+C_{g}\left[\frac{R_{c}^{(0)}}{3 \cdot 4}-\frac{R_{0}^{(1)}}{2}\right] \frac{z^{3}}{2 \cdot 3} \\
& +C_{g}\left[\frac{R_{c}^{(1)}}{2 \cdot 3 \cdot 4}-0.0081184 R_{c}^{(0)}\right] z, \\
& g^{(1)}=C_{g}\left[-\frac{z^{6}}{6}+\frac{z^{5}}{2}-\frac{5}{6} z^{3}+\frac{z^{2}}{2}-\frac{1}{2 \cdot 4 \cdot 7}\right]
\end{aligned}
$$

A constant of integration which appears in $g^{(1)}$ is fixed by using the normalization condition $\int g^{(0)} g^{(1)} d z=0$, which follows from (3.20).

$\left\langle O\left(a^{4}\right)\right\rangle$ :

$$
D^{2} g^{(2)}=g^{(1)}-h^{(1)}
$$

The solvability condition gives

$$
R_{c}^{(1)}=\frac{6060 \cdot 2 \cdot 3 \cdot 4 \cdot 5}{6 \cdot 7 \cdot 8 \cdot 9 \cdot 10 \cdot 11}+\frac{30 \cdot 5}{7}=23.6147
$$

Thus the critical Rayleigh number $R_{c}$ is determined up to $O\left(a^{2}\right)$ in the following form:

$$
R_{c}=R_{c}^{(0)}+a^{2} R_{c}^{(1)}=120+23.6147 a^{2} .
$$

$\left[O\left(\varepsilon^{2}\right)\right]$ : The governing equations at $O\left(\varepsilon^{2}\right)$ are given by (3.16), (3.17), and (3.18). As before, we expand variables in the power series of $a^{2}$.

$$
\begin{gathered}
\theta_{2}^{\mathrm{I}}=\theta_{2}^{\mathrm{I}(0)}+a^{2} \theta_{2}^{\mathrm{I}(1)}+a^{4} \theta_{2}^{\mathrm{II}(2)}+\cdots, \\
\theta_{2}^{\mathrm{II}}=\theta_{2}^{\mathrm{II}(0)}+a^{2} \theta_{2}^{\mathrm{II}(1)}+a^{4} \theta_{2}^{\mathrm{II}(2)}+\cdots, \\
W_{2}^{\mathrm{II}}=W_{2}^{\mathrm{II}(0)}+a^{2} W_{2}^{\mathrm{II}(1)}+a^{4} W_{2}^{\mathrm{II}(2)}+\cdots .
\end{gathered}
$$


$\left\langle O\left(a^{0}\right)\right\rangle:$

$$
\begin{gathered}
D^{2} \theta_{2}^{\mathrm{I}(0)}=0, \\
D^{4} W_{2}^{\mathrm{II}(0)}=0, \\
D^{2} \theta_{2}^{\mathrm{II}(0)}+W_{2}^{\mathrm{II}(0)}=0 .
\end{gathered}
$$

The solutions are

$$
\theta_{2}^{\mathrm{I}(0)}=C \text { (constant), } \quad W_{2}^{\mathrm{II}(0)}=0, \quad \text { and } \theta_{2}^{\mathrm{II}(0)}=0 .
$$

The constant of integration $C$ is set to zero due to the normalization condition. $\left\langle O\left(a^{2}\right)\right\rangle$ :

$$
\begin{gathered}
D^{2} \theta_{2}^{\mathrm{I}(1)}=\frac{1}{2} h^{\prime(0)} g^{(0)}, \\
D^{4} W_{2}^{\mathrm{II}(1)}=0, \\
D^{2} \theta_{2}^{\mathrm{II}(1)}+W_{2}^{\mathrm{II}(1)}=-\frac{1}{2} h^{\prime \prime(0)} g^{(0)},
\end{gathered}
$$

and

$$
\begin{gathered}
W_{2}^{\mathrm{II}(1)}=0, \\
\theta_{2}^{\mathrm{I}(1)}=\frac{5}{2} C_{g}^{2}\left[\frac{z^{5}}{5}-\frac{z^{4}}{2}+\frac{z^{2}}{2}\right]+C_{2}^{\mathrm{I}(1)}, \\
\theta_{2}^{\mathrm{II}(1)}=-\frac{5}{2} C_{g}^{2}\left[\frac{z^{5}}{5}-\frac{z^{4}}{2}+\frac{z^{2}}{2}\right]+C_{3}^{\mathrm{II}(1)} .
\end{gathered}
$$

The constants of integration in Eqs. (A.29) and (A.30) are determined by the solvability condition of the next order energy equation. The normalization condition does not yield useful information in this case because we are considering different modes from $\cos a x$ at this order.

$\left\langle O\left(a^{4}\right)\right\rangle$ :

$$
\begin{gathered}
D^{2} \theta_{2}^{\mathrm{I}(2)}=\frac{1}{2} h^{\prime(1)} g^{(0)}+\frac{1}{2} h^{\prime(0)} g^{(1)}+\frac{1}{2} h^{(0)} g^{\prime(1)}, \\
D^{4} W_{2}^{\mathrm{II}(2)}=4 R_{c}^{(0)}+\frac{1}{P r}\left[h^{(0)} h^{\prime \prime \prime}(0)-h^{(0) \prime} h^{(0) \prime \prime}\right], \\
D^{2} \theta_{2}^{\mathrm{II}(2)}=4 \theta_{2}^{\mathrm{II}(1)}-W_{2}^{\mathrm{II}(2)}-\frac{1}{2} h^{(1) \prime} g^{(0)}-\frac{1}{2} h^{\prime(0)} g^{(1)}+\frac{1}{2} h^{(0)} g^{\prime(1)} .
\end{gathered}
$$

If we included the $R_{1}$ term in the $\varepsilon$ expansion of $R$ (Eq. (3.5)), that term would appear in the right-hand side of Eq. (A.31). But since

$$
\int_{0}^{1} \frac{1}{2} h^{(1)} g^{(0)}+\frac{1}{2} h^{0) \prime} g^{(1)}+\frac{1}{2} h^{(0)} g^{\prime(1)} d z=0
$$

$R_{1}$ would be set to zero after applying the solvability condition. The solvability condition for Eq. (A.33) determines the value of $C_{3}^{\mathrm{II}(1)}$. We omit the detailed expressions of $\theta_{2}^{\mathrm{I}(2)}, W_{2}^{\mathrm{II}(2)}$, and $\theta_{2}^{\mathrm{II}(2)}$ because they are not needed in the derivation of the Landau equation that is valid up to $O\left(a^{2}\right)$.

$\left[O\left(\varepsilon^{3}\right)\right]$ : The governing equations at $O\left(\varepsilon^{3}\right)$ are Eqs. (3.21) and (3.22). We assume the following series expansions in $a^{2}$ :

$$
\Phi_{3}=\Phi_{3}^{(0)}+a^{2} \Phi_{3}^{(1)}+a^{4} \Phi_{3}^{(2)}+\cdots,
$$




$$
\begin{gathered}
\psi_{3}=\psi_{3}^{(0)}+a^{2} \psi_{3}^{(1)}+a^{4} \psi_{3}^{(2)}+\cdots, \\
(d A / d \tau)=a^{4}(d A / d \tau)^{(2)}+\cdots, \\
R_{2}=a^{2} R_{2}^{(1)}+a^{4} R_{2}^{(2)}+\cdots .
\end{gathered}
$$

The flow variables $(\mathbf{u}, \theta)$ become those of linear equations as $a^{2}$ approaches zero $\left(\mathbf{u} \rightarrow \varepsilon u_{1}^{(0)}, \theta \rightarrow \varepsilon \theta_{1}^{(0)}\right.$ as $\left.a^{2} \rightarrow 0\right)$. Therefore, in the expansion of $R_{2}$ (Eq. (A.38)), we have to take $R_{2}^{(0)}$ to be zero.

$\left\langle O\left(a^{0}\right)\right\rangle$ :

$$
\begin{gathered}
D^{2} \psi^{(0)}+\Phi_{3}^{(0)}=0, \\
D^{4} \Phi_{3}^{(0)}=0,
\end{gathered}
$$

and

$$
\Phi_{3}^{(0)}=\psi_{3}^{(0)}=0
$$

$\left\langle O\left(a^{2}\right)\right\rangle$

$$
\begin{gathered}
D^{2} \psi_{3}^{(1)}+\Phi_{3}^{(1)}=0, \\
D^{4} \Phi_{3}^{(1)}=0,
\end{gathered}
$$

and

$$
\Phi_{3}^{(1)}=\psi_{3}^{(1)}=0
$$

$\left\langle O\left(a^{4}\right)\right\rangle$

$$
\begin{gathered}
D^{4} \Phi_{3}^{(2)}=A R_{2}^{(1)} g^{(0)} \\
D^{2} \Psi_{3}^{(2)}+\Phi_{3}^{(2)}=g^{(0)}(d A / d \tau)+A^{3}\left[h^{\prime(0)} \theta_{2}^{\mathrm{II}(1)}+\frac{1}{4} D W_{2}^{\mathrm{II}(2)} g^{(0)}\right. \\
\left.+h^{(0)} D \theta_{2}^{\mathrm{I}(1)}+\frac{1}{2} h^{(0)} D \theta_{2}^{\mathrm{II}(1)}\right]
\end{gathered}
$$

Solving (A.45) with the boundary conditions, we find

$$
\Phi_{3}^{(2)}=A R_{2}^{(1)} C_{g} \frac{1}{2}\left[\frac{z^{4}}{3 \cdot 4}-\frac{z^{3}}{3 \cdot 2}\right]+A R_{2}^{(1)} C_{g} \frac{1}{4 \cdot 3 \cdot 2} z .
$$

The solvability condition for (A.46) gives the following Landau equation governing the evolution of amplitude of the convection cell just above the critical Rayleigh number:

$$
C_{g} \frac{d A}{d \tau}=\frac{R_{2}^{(1)}}{2 \cdot 3 \cdot 4 \cdot 5} A C_{g}-\frac{3}{2} \cdot \frac{25}{2} \cdot \frac{31}{9 \cdot 7 \cdot 5 \cdot 2} A^{3} C_{g}^{3} .
$$

The amplitude of the steady two-dimensional convection roll is given by

$$
\left(A C_{g}\right)^{2}=R_{2}^{(1)} \frac{2 \cdot 4 \cdot 5 \cdot 7 \cdot 9}{2 \cdot 3 \cdot 4 \cdot 5 \cdot 3 \cdot 25 \cdot 31}
$$

and

$$
\sqrt{\left\langle\theta^{2}\right\rangle}=\sqrt{\Delta R(0.0045161) / a^{2}}
$$

where $\Delta R \equiv R-R_{c}$ and $a=\pi / 4$. 


\section{REFERENCES}

[1] C. Canuto, M. Y. Hussaini, A. Quarderoni, and T. A. Zang, Spectral Methods in Fluid Dynamics, Springer-Verlag, New York, 1988

[2] S. Chandrasekhar, Hydrodynamic and Hydromagnetic Stability, Oxford Univ. Press, 1961

[3] P. G. Drazin and W. H. Reid, Hydrodynamic Stability, Cambridge Univ. Press, 1981

[4] E. Jakeman, Convective instability in fluids of high thermal diffusivity, Phys. Fluids 2 (1), 10-14 (1968)

[5] H. Jeffreys, The stability of a layer of fluid heated below, Philos. Mag. (7) 2, 833-44 (1926)

[6] L. Kleiser and U. Schuman, Spectral Simulations of the Laminar-Turbulent Transition Process in Plane Poiseuille Flow, Spectral Methods for Partial Differential Equations, editors: R. G. Voigt, D. Gottlieb, and M. Y. Hussaini, SIAM, 141-163 (1984)

[7] W.V.R. Malkus, and G. Veronis, Finite amplitude cellular convection, J. Fluid Mech. 4, 225-60 (1958)

[8] C. Normand, Y. Pomeau, and M. G. Velarde, Rev. Modern Phys. 49, 581 (1977)

[9] N. Riahi and F. H. Busse, Nonlinear convection in a layer with nearly insulating boundaries, J. Fluid Mech. 96, 243-256 (1980)

[10] A. Schlüter, D. Lortz, and F. Busse, On the stability of steady finite amplitude convection, J. Fluid Mech. 23, 129-44 (1965)

[11] L. Sirovich, H. Tarman, and M. Maxey, Analysis of turbulent thermal convection, Sixth Symposium of Turbulent Shear Flow, Toulouse, 1987

[12] L. Sirovich and H. Park, Turbulent thermal convection in a finite domain: Par I: Theory, Phys. Fluids A, 2 (9), 1649-1658 (1990)

[13] E. M. Sparrow, R. J. Goldstein, and V. K. Jonsson, Thermal instability in a horizontal fluid layer: effect of boundary conditions and non-linear temperature profile, J. Fluid Mech. 18, 513-28 (1964) 